\title{
MODELOS DE SOBREVIVÊNCIA PARA AVALIAÇÃO DE INTOXICAÇÃO POR REPELENTES A BASE DE NEEM EM Trigona spinipes
}

\author{
Luiz Fernando Silva Resende ${ }^{1}$, Carla R G Brighenti ${ }^{12^{*}}$, Deodoro Magno Brighenti ${ }^{2}$, Lívia Mendes Carvalho ${ }^{3}$ \\ 1 Universidade Federal de Lavras, Caixa Postal 3037 - Lavras -MG, 37200-000 \\ ${ }^{2}$ Universidade Federal de São João del-Rei, Campus Tancredo Neves - CTAN Av. Visconde do Rio Preto s/nº, Fábricas, São João del- \\ Rei - MG 36301-360 \\ ${ }^{3}$ Empresa de Pesquisa Agropecuária de Minas Gerais (EPAMIG), Campo Experimental Risoleta Neves, Av. Visconde do Rio Preto s/n \\ , Fábricas, São João del-Rei - MG 36301-360 \\ Autor para correspondência: Carla Regina Guimarães Brighenti, carlabrighenti@ufsj.edu.br
}

RESUMO: A abelha irapuá (Trigona spinipes) é uma espécie de abelha sem ferrão polinizadora de várias espécies vegetais, mas que também tem causado danos a algumas culturas tais como a flor copo-de-leite (Zantedeschia aethiopica). Isto porque, as abelhas ao coletarem o pólen ou resinas nesta espécie, danificam suas inflorescências, deixando-as inviáveis para comercialização. Para evitar este dano uma alternativa é aplicar um repelente natural que não ocasione mortalidade às abelhas, tanto pelo contato direto durante a aplicação do produto por pulverização, quanto por ingestão ao coletar o pólen. Objetivou-se ajustar modelos para avaliação da sobrevivência de abelhas $T$. spinipes, submetidas a bioensaios de intoxicação utilizando diferentes produtos químicos contendo o repelente natural Neem (Azadirachta indica). Foram distribuídas 400 abelhas em gaiolas com grupos de 10 abelhas cada, sendo considerado um delineamento inteiramente casualizado em ensaio fatorial $2 \times 4$, considerando duas vias de intoxicação utilizando três produtos químicos como repelentes e uma testemunha, com cinco repetições cada. Para construção das curvas de sobrevivência foi avaliado o número de abelhas mortas a cada 12 horas considerado como covariáveis a via de intoxicação e o produto químico repelente. Comparou-se os resultados por análise não paramétrica utilizando estimador limite-produto modificado (Turnbull), pelo ajuste por modelo paramétrico de Weibull e pela técnica semiparamétrica de modelos proporcionais de Cox. O melhor ajuste foi obtido pelo modelo semiparamétrico, com valores bem próximos aos obtidos pela estimativa de Turnbull. Não se obteve diferença significativa entre os produtos químicos avaliados ao nível de $5 \%$ de significância. No entanto, conclui-se que a via de intoxicação das abelhas, ou seja, a forma de aplicação do repelente é o fator de maior significância, sendo aguda por contato a via de intoxicação que causa maior mortalidade. Assim, recomenda-se que o horário de aplicação do produto seja diferente do horário de forrageamento da $T$. spinipes.

PALAVRAS-CHAVE:Abelha sem ferrão, modelos de Cox, Azadirachta indica, meliponíneos, polinizadores, inseticida.

\section{SURVIVAL MODELS TO EVALUATE THE INTOXICATION BY REPELLENTS IN Trigonaspinipes}

ABSTRACT: The Irapuá bee (Trigona spinipes) is a stingless bee that pollinates several plant species, but has also caused damage to some crops such as Calla Lily (Zantedeschia aethiopica). It happens because while collecting the pollen, they damage the flowers inflorescence, making them unviable for commercialization. To avoid these damages, an alternative is to apply a natural repellent that does not cause mortality, neither while being sprayed nor by being ingested when the bees collect the pollen. The objective of this study was to adjust models to evaluate the survival of $T$. Spinipes bees, submitted to poisoning bioassays using different chemical products containing the natural Neem repellent (Azadirachtaindica). Four hundred bees were divided into groups of 10 bees each, being considered a completely randomized design in a $2 \times 4$ factorial test, considering two routes of intoxication using three chemicals as repellents and one witness, with five replicates each. To construct the survival curves, it was studied the number of bees killed every 12 hours, and the variables considered were the route of intoxication and the chemical repellent used. The results by non-parametric analysis using modified limit-product estimator 
(Turnbull) were compared by setting Weibull parametric model and semi-parametric technique of Cox proportional models. The best result was obtained by the semi-parametric model, with values very close to those obtained by Turnbull's estimation. There was no significant difference between the evaluated chemicals at the $5 \%$ level of significance. However, it is concluded that the bee's route of intoxication is the form in which the repellent is applied, causing mortality by contact. Thus, the application of the repellent should be different from the T. Spinipes foraging schedule.

KEY WORDS: Stingless bee, Cox models, Azadirachta indica, meliponines, pollinators, insecticide.

\section{INTRODUÇÃO}

Abelhas Trigona spinipes são insetos sociais de colônias perenes com centenas a milhares de operárias. A abelha adulta apresenta coloração preta, mandíbulas desenvolvidas, asas transparentes, com ferrão atrofiado, portanto são chamadas de abelhas sem ferrão. Medem cerca de 5 à 7,5 mm de comprimento e enrolam-se nos cabelos das pessoas quando são perturbadas, sendo conhecida também como abelha cachorro ou irapuá (Zucchi et al., 1993).

É descrita como agente polinizador de diversas culturas, podendo ser utilizada inclusive como agente polinizador comercial (Sanches et al., 2001). No entanto são também consideradas pragas em outras culturas, principalmente frutíferas (Coelho et al., 2008) e também de ornamentais como rosas, crisântemos, camélias e copo de leite, provocando abertura das pétalas e danos nos botões florais. $\mathrm{Na}$ flor copo-de-leite (Zantedeschia aethiopica) a abelha irapuá, ao coletar o pólen danifica a inflorescência da flor deixando-a inviável para comercialização (Carvalho et al., 2009). Moura et al. (2017) observaram também a $T$. spinipes predando a parte apical do caule jovem de Khayaivorensis (Mogno africano), causando atrofia e brotação, provocando ramificações que irão depreciar o fuste se não manejado. Muitos são os trabalhos encontrados relatando os prejuízos causados por abelhas $T$. spinipes em diversas culturas, tais como banana, uva, manga,citrus (Agrofit, 2019) e maracujazeiro amarelo (Boiça Junior et al., 2014), porém poucas são as estratégias de controle.

Chiaradia et al. (2003) relatam como estratégia pulverizar as plantas atacadas com substâncias que possuem cheiros fortes e desagradáveis, como a creolina. Porém, não há eficácia comprovada cientificamente relatada acerca dessas práticas bem como não é possível qualificar ou quantificar os danos causados às colônias. Outros autores ainda sugerem 0 controle cultural realizado pela destruição dos ninhos (Brasil,2015).

É importante ressaltar quenão existe um produto de controle químico registrado, no Sistema de Agrotóxicos Fitossanitários (Agrofit, 2019), especificamente para a $T$. spinipesna cultura de copode-leite, sendo indicado no sistema apenas o uso de inseticidas no geral. Entretanto, deve-se ressaltar que cerca de $60 \%$ dos inseticidas comercializados no Brasil apresentam toxicidade para abelhas (Pinheiro e Freitas, 2010).

As abelhas podem ser afetadas por inseticidas através das duas principais vias de intoxicação: pulverização (toxicidade aguda por contato) ou ingestão (toxicidade oral crônica) e, seus efeitos variam conforme as concentrações e as doses utilizadas, modo de intoxicação e tempo de exposição ao inseticida (Rossi et al., 2013).

Para a produção de defensivos agrícolas, os óleos essenciais ocupam um lugar de destaque, uma vez que apresentam propriedades fungicidas e inseticidas contra pragas que causam prejuízos aos agricultores, acarretando perdas na produtividade e qualidade das culturas (Simões et al., 2004). Dentre os óleos essenciais, o produzido a partir da planta Neem (Azadirachta indica) tem grande destaque.

O Neem (Nim) é uma planta que pertence à família do mogno e do cedro. São árvores de grande porte, podendo atingir até $30 \mathrm{~m}$ de altura e $2,5 \mathrm{~m}$ de diâmetro. Além de fornecer madeira, é muito conhecida por suas propriedades medicinais e terapêuticas encontradas nas sementes, folhas e casca. É bastante utilizada na agricultura, no combate a lagartas e pragas como nematóides, fungos e bactérias. É uma das plantas de maior potencial no controle de pragas, atuando sobre $95 \%$ dos insetos nocivos (Previero et al., 2010).

O óleo de Neem possui propriedades fungicidas e bactericidas, além de ser um óleo que é facilmente disponível para seu uso como uma erva medicinal de 
baixo custo (Santos el al., 2017).É relatado seu uso em alguns estudos com polinizadores, a maior parte deles citando a abelha Apis mellifera e negligenciando outras espécies importantes de polinizadores (Barbosa et al., 2015). Boeke et al. (2004) e Nisbet et al.(2000) avaliaram a segurança do uso de pesticidas derivados de Neem como inseticida, incentivando sua aplicação. O óleo de Neem aplicado topicamente ofereceu proteção significativa das abelhas contra a infestação por Acarapis woodi, embora não tenha resultado em mortalidade direta do ácaro (Melathopoulos et al., 2000). Finalmente é importante ressaltar que $0 \mathrm{~A}$. indica ainda não é registrado no Brasil para uso agrícola (Freitas e Pinheiro, 2012).

Ao realizar experimentos com abelhas em laboratórios as características de interesse são analisadas em grupos de abelhas confinadas em gaiolas e, normalmente, se avalia a mortalidade em intervalos de tempo pré-determinados (Pirk et al., 2013). Isto ocorre por se tratar de insetos que vivem em sociedade e, para evitar alterações fisiológicas e comportamentais devem ser mantidos sempre em grupo. Já que os dados são coletados em intervalos de tempo, não se conhece o tempo exato de ocorrência da característica de interesse, neste caso a morte do inseto. Assim, tem-se uma informação incompleta denominada de dado "censurado" e por isso faz-se necessária a utilização de técnicas estatísticas de análise de sobrevivência.

Os modelos de sobrevivência podem ser estimados de forma não paramétrica seguindo, por exemplo, o estimador limite-produto modificado de Turnbull, paramétrica, realizando o ajuste por modelos como Weibull ou log-normal, ou ainda semiparametricos como os modelos de risco proporcionais de Cox, tal como utilizado por Moncharmont et al. (2003).

Diante do exposto, objetivou-se ajustar modelos para avaliação da sobrevivência de abelhas $T$. spinipes, submetidas a formas de aplicação do repelente natural Neem(Azadirachta indica).

\section{MATERIAL E MÉTODOS}

Os adultos de abelhas irapuá foram obtidos diretamente da colônia localizada em condições naturais no Campus Tancredo de Almeida Neves da Universidade Federal de São João del Rei, MG (UFSJ). Para realizar a captura dos indivíduos foi projetado um coletor de abelhas feito com cano de PVC com uma das extremidades fechadas com duas camadas de tecido organza, visando evitar fuga por cortes provocados por suas mandíbulas. $O$ capturador foi posicionado na entrada da colônia e à medida que iam saindo para forrageamento ou defesa do ninho ficaram presas no coletor. Assim que uma quantidade adequada de abelhas foi capturada, procedeu-se 0 fechamento com um tampão, repetindo-se o processo se necessário.

Posteriormente foram transportadas para 0 Laboratório de Praticas Apícolas do Departamento de Zootecnia. Para montagem das unidades experimentais foram utilizadas gaiolas de PVC cilíndricas com 10 $\mathrm{cm}$ de diâmetro por $5 \mathrm{~cm}$ de altura, revestidas com tecido duplo de organza para contagem dos insetos. As abelhas foram anestesiadas por abaixamento de temperatura e separadas em grupos de 10 indivíduos para cada unidade experimental e mantidas em câmara climatizada a $30 \pm 2^{\circ} \mathrm{C}$, UR $70 \pm 10 \%$ e fotoperíodo de 12 horas.

0 estudo de toxicidade realizou-se em dois bioensaios em abelhas adultas de $T$. spinipes: aguda por contato e oral crônica utilizando três diferentes produtos químicos repelentes contendo NeemFertbom $^{\circledR}$, I Go $^{\circledR}$ e o extrato pirolenhoso Biopirol ${ }^{\circledR}$, além do tratamento testemunha apenas com a solução aquosa de sacarose, totalizando oito tratamentos, sendo utilizadas cinco repetições de cada.

Para o bioensaiode toxicidade oral crônica, foi preparada uma solução aquosa à $50 \%$ de sacarose $\mathrm{PA}$, após o preparo foi feita a contaminação do alimento com a dosagem de $5 \mathrm{~mL} / \mathrm{L}$ do repelente conforme recomendação dos fabricantes. Estas foram colocadas no interior das gaiolas em uma tampa plástica de três centímetros de diâmetro por $1,5 \mathrm{~cm}$ de altura, contendo um chumaço de algodão para evitar afogamento. Todos os tratamentos receberam água ad libitum em tampa plástica de mesma característica.

Para o bioensaio de toxicidade aguda por contato foram utilizados pulverizadores manuais para a aplicação dos inseticidas naturais. A taxa de aplicação média por pulverizador foi de $8,4 \times 10-4 \mathrm{~mL} / \mathrm{cm}^{2}$. Em cada repetição foram realizadas dez borrifadas, a uma distância de $\pm 25 \mathrm{~cm}$ do alvo (Carvalho, 2009). Os insetos foram alimentados com solução aquosa à $50 \%$ de sacarose PA e água em recipientes semelhantes ao bioensaio de toxicidade oral. 
Avaliou-se o número de abelhas mortas a cada 12 horas, sendo esta a variável resposta,ou seja, 0 tempo ti até a ocorrência da morte da abelha, gerando dados com censura intervalar. 0 experimento teve duração de 20 dias (480 horas), após isso o tempo de mortalidade foi considerado como censurado. Não foi avaliado o consumo nos bioensaios.

Para a análise de sobrevivência utilizouse inicialmente 0 estimador limite-produto, também denominado estimador não paramétrico de KaplanMeier (KM), definido como:

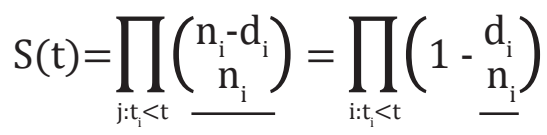

em que $t_{1}<t_{2}<\cdots<t_{k}$, são $k$ os tempos distintos $\mathrm{e}$ ordenados de morte, $d_{i} 0$ número de mortes em $t_{i}, \mathrm{i}=$ $1, \ldots, k$, e o número de abelhas que não morreram e não foram censuradas até 0 instante imediatamente anterior a. Dados que as respostas do tempo de vida são obtidas em sua totalidade com censura intervalar, ocorreram com frequência os empates, ou seja, número de mortes considerando o mesmo tempo de ocorrência. Na ocorrência de empates, Giolo e Colossimo (2006), sugerem a utilização do estimador de Turnbull que é uma adaptação do método $\mathrm{KM}$ e corresponde a um processo iterativo que considera uma ponderação de cada ocorrência de mortalidade nos intervalos no instante $t_{i}$ em relação ao $t_{i-1}$.

Para a modelagem paramétrica foi inicialmente selecionada a melhor distribuição a ser ajustada ao tempo de vida considerando as distribuições exponencial, Weibull e log-normal e posteriormente considerado 0 ajuste do modelo de regressão, incluindo as covariáveis. No caso do modelo Weibull tem-se que, para a variável Tempo ( $\mathrm{t}$ ) condicional ao vetor de covariáveis $\mathrm{x}$, a função de sobrevivência correspondente é $S(t \mid x)=\exp \{-$ $\left.\left(\exp \left\{x^{\prime} \beta\right\}\right)^{\delta}\right\}$ em que $x^{\prime}$ é a matriz de covariáveis; $\beta 0$ parâmetro de forma da distribuição e corresponde ao vetor de parâmetros associado às covariáveis estimados pelo método da máxima verossimilhança. Salienta-se que se $\delta=1$ a modelagem paramétrica passa a ser a distribuição exponencial (Archer, 2014).

0 uso de um modelo paramétrico pode gerar resultados viesados ou inconsistentes. Assim, para comparação, considerou-se ainda 0 modelo semiparamétrico de riscos proporcionais de Cox que propõe a modelagem, na presença de covariáveis, por meio da função de risco dada por $\lambda(t \mid x)=\lambda_{0}(t) \exp \left\{x^{\prime} \beta\right\}$ (Maia, 2016). Sua vantagem é modelar e testar hipóteses independente da forma da distribuição do tempo de sobrevivência e o método de estimação de parâmetros é baseado na verossimilhança parcial (Lin, 1994). As análises foram adaptadas para utilização do pacote icenReg do software $R$ (R Team, 2018).

Consideraram-se como covariáveis a via de intoxicação $x_{1}$, sendo $x_{1}=0$ para oral crônica e $x_{1}=1$ quando a intoxicação ocorreu porcontato, e o produto químico do repelente como covariável $x_{2}$. Para 0 produto químico do repelente, considerou-se quatro categorias: $x_{2}=0$ para testemunha, na ausência de repelente, $x_{1}=1$ para a 0 produto químico Fertbom ${ }^{\circledR}$, $x_{2}=2$ para I Go ${ }^{\circledR}$, e $x_{2}=3$ para Biopirol ${ }^{\circledR}$.

Para selecionar o melhor modelo foram utilizadas técnicas gráficas, seja pela comparação da estimativa Turnbull com os modelos paramétricos ou, pelo $Q-Q$ Plot (Giolo e Colossimo, 2006). Para evitar componente subjetivo na interpretação gráfica, foi utilizado também o critério de informação de AIC dado por $A I C=-2 \log \left(L_{q}\right)+$ $2 p$ em que $L_{q}$ é a função de máxima verossimilhança do modelo e $p$ é o número de covariáveis.

Ressalta-se que os dados de sobrevivência das abelhas não podem ser estudados por meio de técnicas de regressão múltipla devido à distribuição não normal dos tempos de sobrevivência, presença de censura, e as covariáveis dependentes do tempo. No entanto, se considerarmos apenas um tempo fixo, ou seja, uma resposta univariada, como realizado tradicionalmente na área experimental, poderia ser considerado um delineamento inteiramente casualizado em ensaio fatorial $2 \times 4$ ( 2 vias de intoxicação e 3 produtos químicos e testemunha). Mas neste caso, a informação da variação temporal da mortalidade seria perdida. Para fins de comparação dessa situação foi analisada determinandose o tempo fixo para obtenção da taxa de mortalidade igual $48 \mathrm{~h}$ e $96 \mathrm{~h}$, conforme recomendações para testes de toxicidade em abelhas (Medrzycki et al., 2013).

\section{RESULTADOS E DISCUSSÃO}

A intoxicação aguda por contato ocasionou maior mortalidade até 288 horas, a partir da qual restavam menos de $10 \%$ de abelhas no experimento. É evidenciada a maior diferença dos métodos de intoxicação nas primeiras 48 horas, quando o método por contato se mostra mais agressivo (Tabela 1). 
Tabela 1. Sobrevivência $(S(t))$ de abelhas Trigona spinipes em função de diferentes vias de intoxicação do repelente Neem ( $x_{1}=0$ intoxicação via oral e $x_{1}=1$ intoxicação via contato) e suas diferenças di em função do tempo t. Temperatura de $30 \pm 2^{\circ} \mathrm{C}$, UR $70 \pm 10 \%$ e fotoperíodo de 12 horas. UFSJ, São João del Rei-MG, 2018.

\begin{tabular}{|c|c|c|c|c|c|c|c|}
\hline \multirow{2}{*}{$t_{i}$} & \multicolumn{2}{|c|}{$S\left(t_{i} \mid x_{1}\right)$} & \multirow{2}{*}{$d_{i}$} & \multirow{2}{*}{$t_{i}$} & \multicolumn{2}{|c|}{$\mathbf{S}\left(\mathrm{t}_{\mathrm{i}} \mid \mathrm{x}_{1}\right)$} & \multirow{2}{*}{$d_{i}$} \\
\hline & $x_{1}=0$ & $x_{1}=1$ & & & $x_{1}=0$ & $x_{1}=1$ & \\
\hline 0 & 1,000 & 1,000 & 0,000 & 228 & 0,263 & 0,113 & 0,149 \\
\hline 12 & 0,975 & 0,820 & 0,155 & 240 & 0,242 & 0,098 & 0,144 \\
\hline 24 & 0,939 & 0,675 & 0,264 & 252 & 0,222 & 0,093 & 0,129 \\
\hline 36 & 0,904 & 0,655 & 0,249 & 264 & 0,187 & 0,077 & 0,110 \\
\hline 48 & 0,854 & 0,624 & 0,230 & 276 & 0,157 & 0,072 & \\
\hline 60 & 0,753 & 0,577 & 0,176 & 288 & 0,101 & 0,062 & 0,039 \\
\hline 72 & 0,722 & 0,557 & 0,165 & 300 & 0,066 & 0,052 & 0,014 \\
\hline 84 & 0,682 & 0,536 & 0,146 & 312 & 0,056 & 0,046 & 0,010 \\
\hline 96 & 0,621 & 0,469 & 0,152 & 324 & 0,051 & 0,046 & 0,005 \\
\hline 108 & 0,596 & 0,459 & 0,137 & 336 & 0,035 & 0,036 & $-0,001$ \\
\hline 120 & 0,545 & 0,428 & 0,118 & 372 & 0,035 & 0,036 & $-0,001$ \\
\hline 132 & 0,485 & 0,351 & 0,134 & 384 & 0,030 & 0,036 & $-0,006$ \\
\hline 144 & 0,414 & 0,268 & 0,146 & 396 & 0,025 & 0,036 & $-0,011$ \\
\hline 156 & 0,394 & 0,247 & 0,147 & 408 & 0,025 & 0,026 & $-0,001$ \\
\hline 168 & 0,374 & 0,222 & 0,152 & 420 & 0,015 & 0,021 & $-0,005$ \\
\hline 180 & 0,354 & 0,196 & 0,158 & 432 & 0,010 & 0,021 & $-0,011$ \\
\hline 192 & 0,278 & 0,165 & 0,113 & 444 & 0,010 & 0,015 & $-0,005$ \\
\hline 204 & 0,273 & 0,129 & 0,144 & 456 & 0,010 & 0,010 & 0,000 \\
\hline 216 & 0,263 & 0,119 & 0,144 & 480 & 0,010 & 0,010 & 0,000 \\
\hline
\end{tabular}

Nos tempos 24, 36 e 48 horas as diferenças entre os métodos de intoxicação são as mais altas, sendo de $26,4 \%, 24,9 \%$, e $23,0 \%$, respectivamente. Até as $276 \mathrm{~h}$ de observação, a mortalidade de abelhas submetidas à intoxicação por contato teve diferença da intoxicação oral variando entre $8 \%$ e $18 \%$. Após este período os dois métodos tornaram-se praticamente semelhantes, restando aproximadamente, apenas $10 \%$ dos indivíduos. Xavier et al. (2015) conclui que a intoxicação oral do repelente causa menor dano as abelhas Trigona spinipes do que quando o mesmo é administrado via contato, principalmente nas primeiras 72 horas.

Considerando as curvas de sobrevivência com a covariávelproduto químico do repelente nas diferentes vias de intoxicação, percebe-se que a variação de mortalidade inicial entre os diferentes produtos químicos e a testemunha é reduzida, sendo mais acentuada apenas após 180 horas de experimento. Entre as duas vias de intoxicação não há também diferença de comportamento entre os produtos químicos (Figura 1a e 1b).

Para ajuste paramétrico dos erros, fez-se necessário, avaliar inicialmente a melhor distribuição. Para tal, testou-se através de comparação com o ajuste paramétrico, as distribuições exponencial, Weibull e log-normal, obtendo-se as estimativas apresentadas na Tabela 2 e seus respectivos valores de AIC. Entre os modelos candidatos o que apresentou menor valor de AIC foi o de Weibull. 0 mesmo resultado pode ser obtido ao avaliar os gráficos ajustados comparados com o ajuste não paramétrico de Turnbull (Figura 2). Quanto ao ajuste obtido pela distribuição de Weibull,este é o que mais se aproxima da estimativa de Turnbull. Por meio do $Q-Q$ Ploto que mais se aproxima de reta $x=y$ é também referente à distribuição de Weibull (Figura 3). Tal resultado está de acordo com o obtido por Brighenti et al. (2017) ao trabalhar dietas para abelhas Apis mellifera. Assim, para a modelagem considerando as covariáveis utilizou-se o modelo de regressão de Weibull.

Pela análise do parâmetro $\alpha$ verifica-se que em $t_{i}=140,1$ a mortalidade atinge $63,21 \%$, ou seja, $S(140,1)=36,79 \%$. Nota-se na Figura 2 que o ajuste fica muito próximo das estimativas de Turnbull, porém, não tão satisfatório entre $t_{i}=72$ e $t_{i}=132$. Um modelo semiparamétrico foi ajustado para obter resultado melhor que o ajuste paramétrico, principalmente no intervalo mencionado anteriormente. 0 modelo semiparamétrico ajustado foi igual a $S(t)=\left[S_{0}(t)\right]^{\exp \{0,3154\}}$. A curva de sobrevivência semiparamétrica, ajustada sem considerar as covariáveis, pelo modelo de regressão discreto tem maior proximidade com a estimativa de Turnbull (Figura 4). 
Tabela 2. Estimativas dos modelos paramétricos para sobrevivência de abelhas Trigona spinipes. Temperatura de $302^{\circ} \mathrm{C}$, UR $7010 \%$ e fotoperíodo de 12 horas. UFSJ, São João del Rei-MG, 2018.

\begin{tabular}{|c|c|c|c|c|}
\hline Distribuição & $S(t)$ & \multicolumn{2}{|c|}{ Parâmetros estimados } & $\mathrm{AIC}$ \\
\hline Exponencial & $S(t)=\exp \left\{-\left(\frac{t}{\alpha}\right)\right\}$ & \multicolumn{2}{|c|}{$a=134,3$} & 2670 \\
\hline Weibull & $S(t)=\exp \left\{-\left(\frac{t}{\alpha}\right)^{y}\right\}$ & $\alpha=140,1$ & $\gamma=1,141$ & 2678 \\
\hline Log-normal & $S(t)=\phi\left(\frac{-\log (t)+\mu}{\sigma}\right)^{2}$ & $\mu=4,460$ & $\sigma=1,089$ & 2761 \\
\hline
\end{tabular}

Tabela 3. Estimativas obtidas para o modelo de regressão paramétrico de sobrevivência $(S(t))$ de abelhas Trigona spinipes. Temperatura de $302^{\circ} \mathrm{C}$, UR 70 10\% e fotoperíodo de 12 horas. UFSJ, São João del Rei-MG, 2018.

\begin{tabular}{cccccc}
\hline Modelo & Covariáveis & Estimativa & Erro padrão & Wald & $p$-valor \\
\hline 1 & Nenhuma & - & - & - & - \\
\hline 2 & $x_{1}=1$ & $\beta_{1}=1,36600$ & 0,1011 & 3,268 & 0,00108 \\
\hline \multirow{3}{*}{3} & $x_{2}=1$ & $\beta_{1}=0,30660$ & 0,1431 & 2,143 & 0,03213 \\
& $x_{2}=2$ & $\beta_{2}=0,07501$ & 0,1423 & 0,527 & 0,59810 \\
& $x_{2}=3$ & $\beta_{3}=-0,29050$ & 0,1442 & $-2,015$ & 0,04393 \\
\hline \multirow{3}{*}{4} & $x_{1}=1$ & $\beta_{1}=0,31920$ & 0,1024 & 3,119 & 0,00182 \\
& $x_{2}=1$ & $\beta_{2}=0,32100$ & 0,1433 & 2,240 & 0,02511 \\
& $x_{2}=2$ & $\beta_{3}=0,03288$ & 0,1429 & 0,230 & 0,81800 \\
& $x_{2}=3$ & $\beta_{4}=-0,26910$ & 0,1444 & $-1,864$ & 0,06232 \\
\hline & $x_{1}=1$ & $\beta_{1}=0,34800$ & 0,1999 & 1,741 & 0,08173 \\
& $x_{2}=1$ & $\beta_{2}=0,24250$ & 0,2001 & 1,212 & 0,22540 \\
& $x_{2}=2$ & $\beta_{3}=0,33990$ & 0,2002 & 1,698 & 0,08948 \\
& $x_{2}=3$ & $\beta_{4}=-0,39040$ & 0,2015 & $-1,938$ & 0,05261 \\
& $x_{1}=1^{*} x_{2}=1$ & $\beta_{5}=0,17930$ & 0,2822 & 0,635 & 0,52530 \\
& $x_{1}=1^{*} x_{2}=2$ & $\beta_{6}=-0,54930$ & 0,2834 & $-1,938$ & 0,05263 \\
& $x_{1}=1^{*} x_{2}=3$ & $\beta_{7}=0,2658$ & 0,2864 & 0,928 & 0,35350 \\
\hline
\end{tabular}

Tabela 4. Modelos de regressão semiparamétrico de sobrevivência $(\mathrm{S}(\mathrm{t}))$ de abelhas Trigona spinipes. Temperatura de $302^{\circ} \mathrm{C}$, UR $7010 \%$ e fotoperíodo de 12 horas. UFSJ, São João del Rei-MG, 2018.

\begin{tabular}{|c|c|}
\hline Modelo & $\hat{\mathbf{S}}(\mathrm{t})$ \\
\hline 1 & $\exp \left\{-\left(\frac{t}{a}\right)^{y}\right\}$ \\
\hline 2 & $\exp \left\{-\left(\frac{t}{a+\beta_{1}{ }^{*} x_{1}}\right)^{n}\right\}$ \\
\hline 3 & $\exp \left\{-\left(\frac{t}{\alpha+\beta_{2}{ }^{*} x_{2}}\right)^{n}\right\}$ \\
\hline 4 & $\exp \left\{-\left(\frac{t}{a+\beta_{1}{ }^{*} x_{1}+\beta_{2}{ }^{*} x_{2}}\right)^{n}\right\}$ \\
\hline 5 & $\exp \left\{-\left(\frac{t}{\alpha+\beta_{1}{ }^{*} x_{1}+\beta_{2}{ }^{*} x_{2}+\left(\beta_{1}{ }^{*} x_{1}\right)\left(\beta_{2}{ }^{*} x_{2}\right)}\right)^{\eta}\right\}$ \\
\hline
\end{tabular}


Figura 1. Curva se Sobrevivência $(\mathrm{S}(\mathrm{t}))$ de abelhas Trigona spinipes em função dos produtos químicos de diferentes repelentes Neem para as duas vias de intoxicação. Temperatura de $30 \pm 2^{\circ} \mathrm{C}$, UR $70 \pm 10 \%$ e fotoperíodo de 12 horas. UFSJ, São João del Rei-MG, 2018.

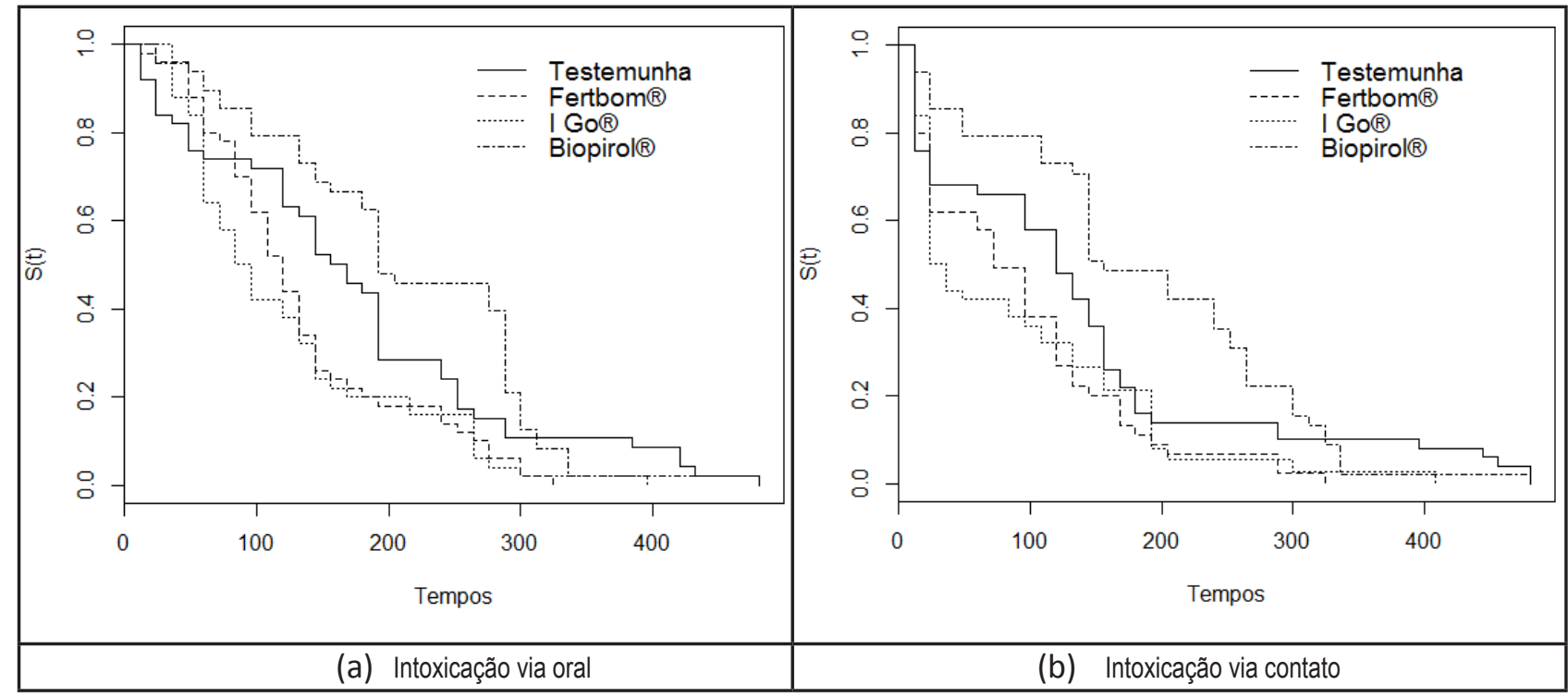

Figura 2. Comparação entre os modelos paramétricos para sobrevivência de abelhas Trigona spinipes e as estimativas de Turnbull. Temperatura de $30 \pm 2^{\circ} \mathrm{C}$, UR $70 \pm 10 \%$ e fotoperíodo de 12 horas. UFSJ, São João del Rei-MG, 2018.

Exponencial



Weibull

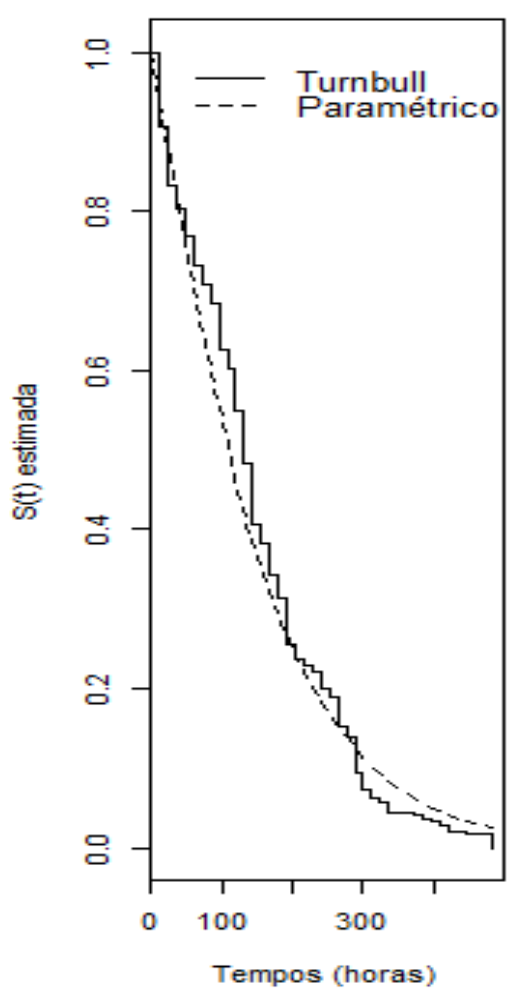

Log-Normal

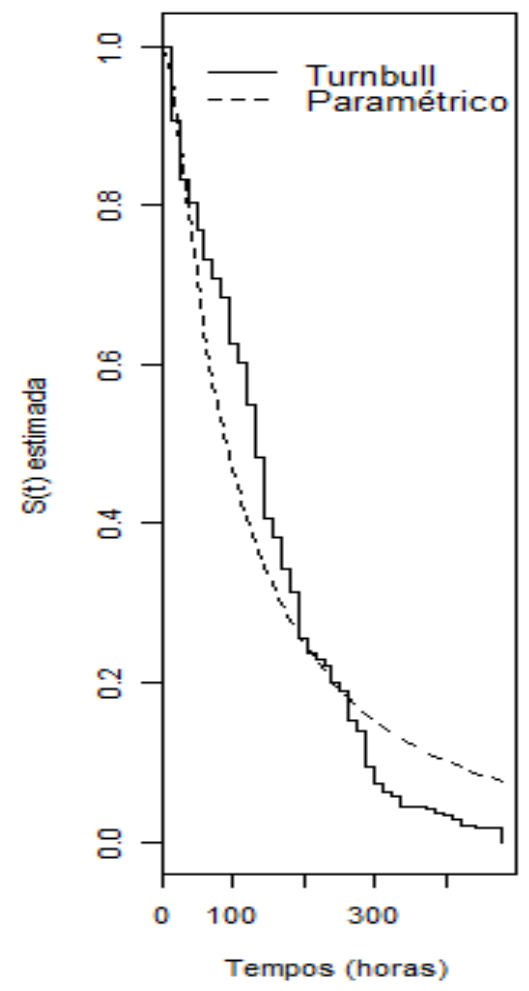


Figura 3. QQPlot entre os modelos paramétricos e as estimativas de Turnbull para sobrevivência de abelhas Trigona spinipes. Temperatura de $30 \pm 2^{\circ} \mathrm{C}$, UR $70 \pm 10 \%$ e fotoperíodo de 12 horas. UFSJ, São João del Rei-MG, 2018.

Exponencial

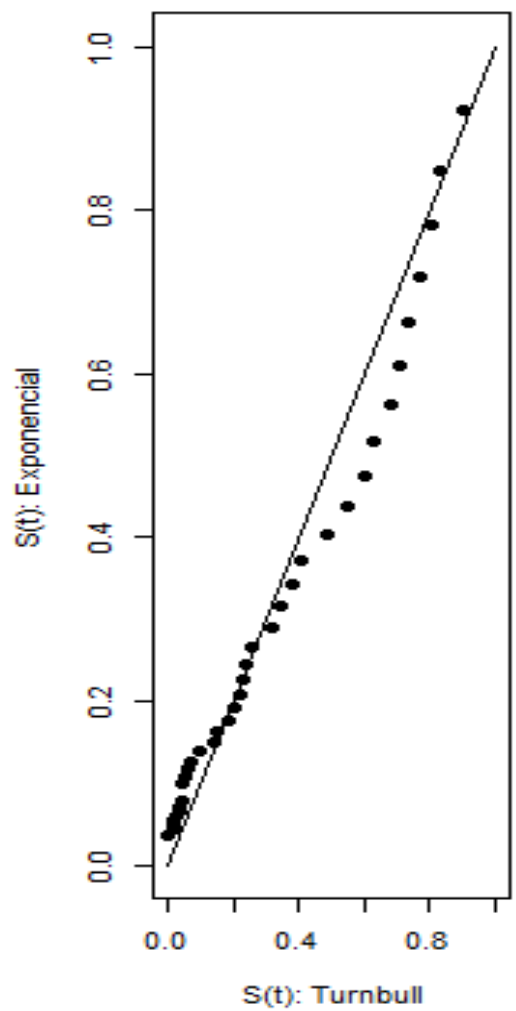

Weibull

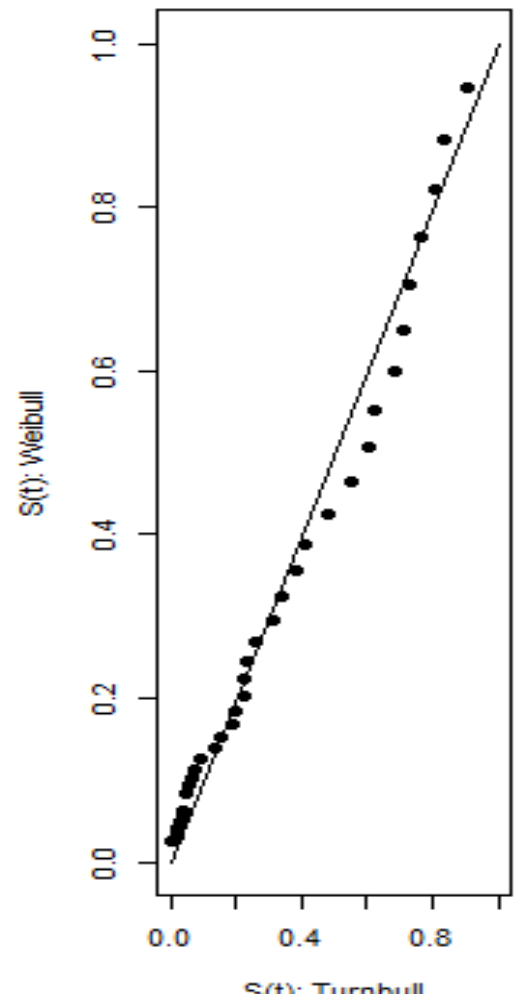

Log-normal

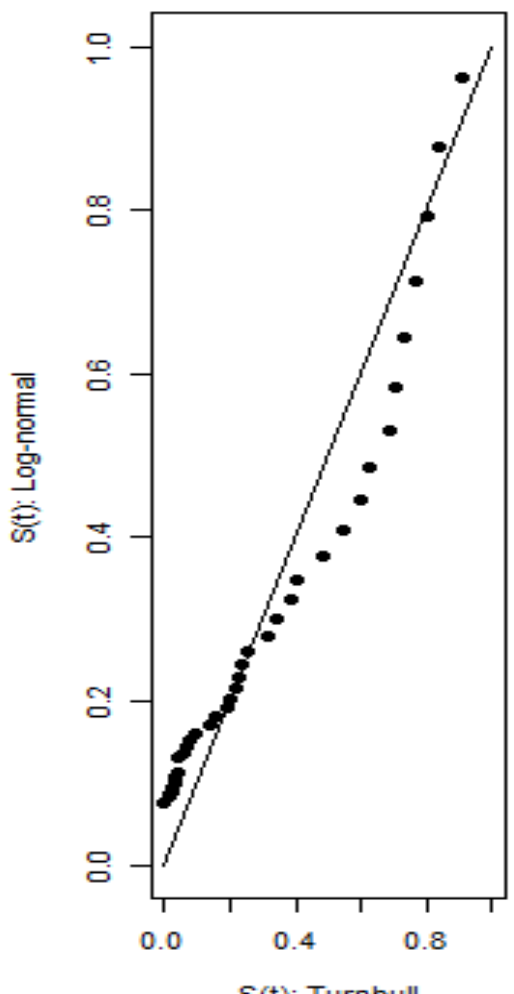

$S(t)$ : Turnbull

Figura 4. Curva de Sobrevivência semiparamétrica $S(t)=\left[S_{0}(t)\right]^{\exp \{0,3154\}}$ de abelhas Trigona spinipes submetidas ao repelente Neem. Temperatura de $30 \pm 2^{\circ} \mathrm{C}$, UR $70 \pm 10 \%$ e fotoperíodo de 12 horas. UFSJ, São João del ReiMG, 2018.

\section{Weibull}

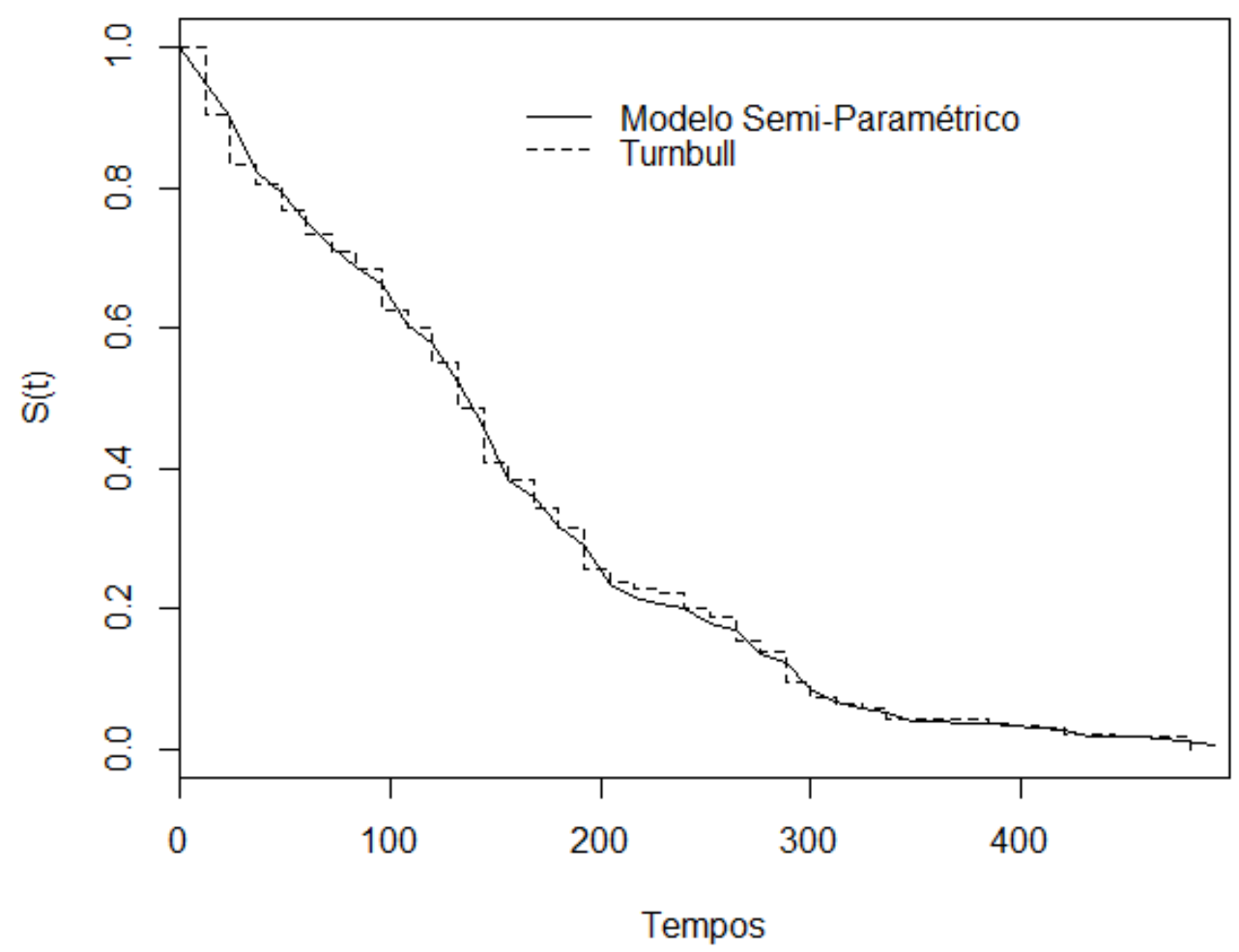


Considerando as covariáveis $\mathrm{x}_{1}$, referente a via de intoxicação, e $x_{2}$, os produtos químicos do repelente, no modelo de regressão Weibull, obtevese os resultados das estimativas dos parâmetros apresentados na Tabela 3, para os modelos considerando as covariáveis e interação, conforme a Tabela 4.

Considerando os valores-p de logverossimilhança, o modelo 2 , com a inclusão da covariável via de intoxicação $\left(x_{1}\right)$, é o único na qual há evidência estatísticas, a $5 \%$ de significância, em que os parâmetros de forma $(\gamma)$ e escala ( $\alpha$ ) estimados são respectivamente 1,142 e 150,2. A vantagem do modelo paramétrico reside no fato de permitir a interpretação dos coeficientes. Assim, o valor positivo obtido para o parâmetro $\beta_{1}=1,366$, implica que as abelhas submetidas a intoxicação via oral $\left(x_{1}=0\right)$ apresentam probabilidade de sobrevivência maior do que as submetidas a intoxicação por contato $\left(x_{1}=1\right)$, o que pode ser observado claramente nas curvas de sobrevivência estimadas (Figura 5). É possível também notar um distanciamento entre as vias de intoxicação logo nas primeiras horas e após aproximadamente 140 horas de experimento. 0 mesmo não ocorre no tempo intermediário e por isso, realizou-se também o ajuste semiparamétrico, considerando também apenas a covariável $\mathrm{x} 1$, na qual ajustou-se o modelo: $S(t)=\left[S_{0}(t)\right]^{\exp \left\{0,21653^{*} \times 1\right\}}$, que corresponde a curva de sobrevivência apresentada na Figura 6. A avaliação da toxicidade em longo prazo para abelhas de exposição crônica a pesticidas por meio de um modelo de risco proporcional revelou que a analise não deve negligenciar a variação temporal da mortalidade.

Figura 5. Curva de Sobrevivência $S(t)=\exp \left\{-\left(\frac{t}{150,2+1,366^{*} x_{1}}\right) 1,142\right\}$ de abelhas Trigona spinipes em função de diferentes vias de intoxicação do repelente Neem, sendo $x_{1}=0$ intoxicação via oral e $x_{1}=1$ intoxicação via contato. Temperatura de $30 \pm 2^{\circ} \mathrm{C}$, UR $70 \pm 10 \%$ e fotoperíodo de 12 horas. UFSJ, São João del Rei-MG, 2018.

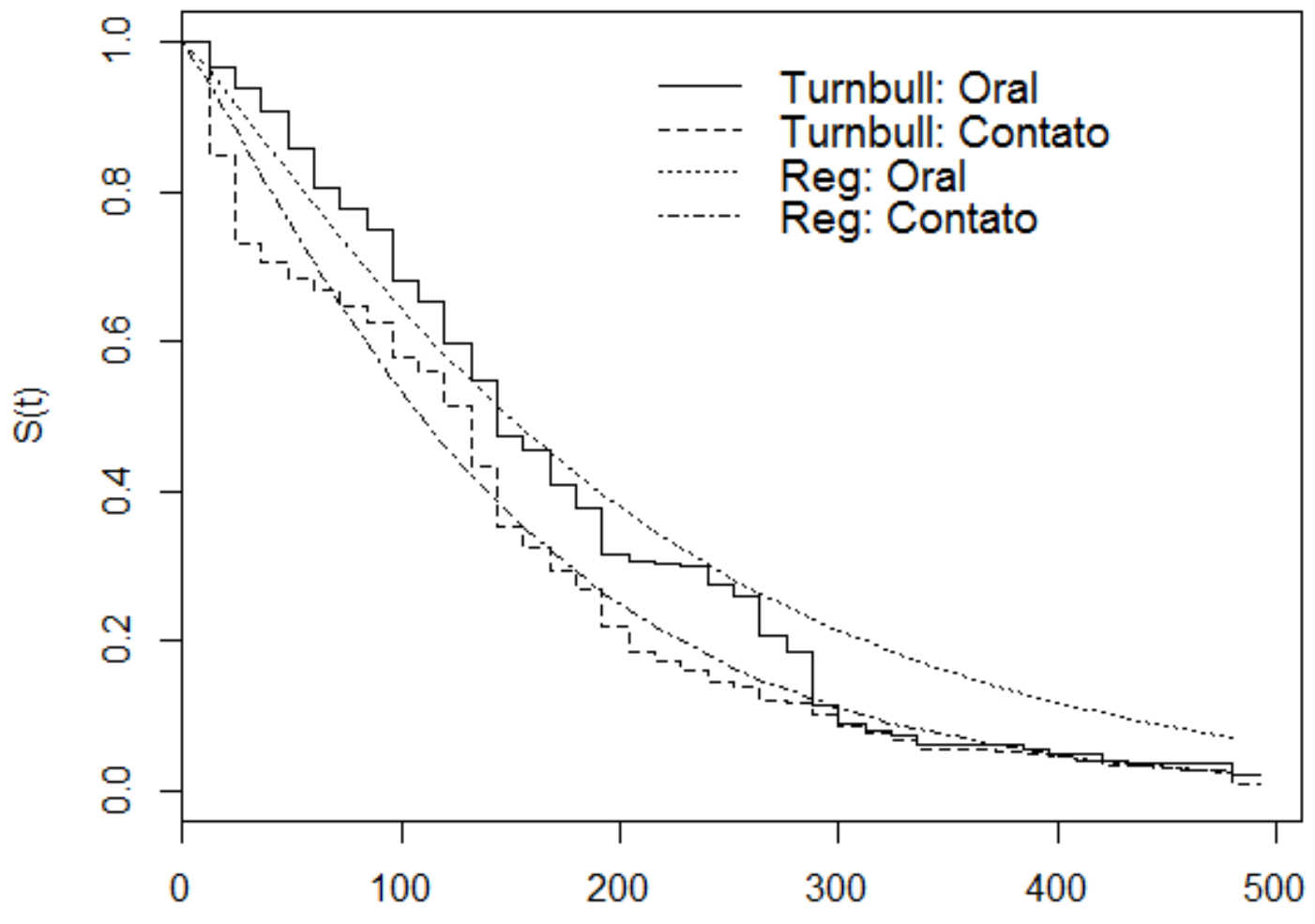

Tempos 
Figura 6. Modelo de Cox de sobrevivência das abelhas Trigona spinipes em função de diferentes vias de intoxicação do repelente Neem, sendo $x_{1}=0$ intoxicação via oral e $x_{1}=1$ intoxicação via contato. Temperatura de 30 $2 \pm{ }^{\circ} \mathrm{C}$, UR $70 \pm 10 \%$ e fotoperíodo de 12 horas. UFSJ, São João del Rei-MG, 2018.

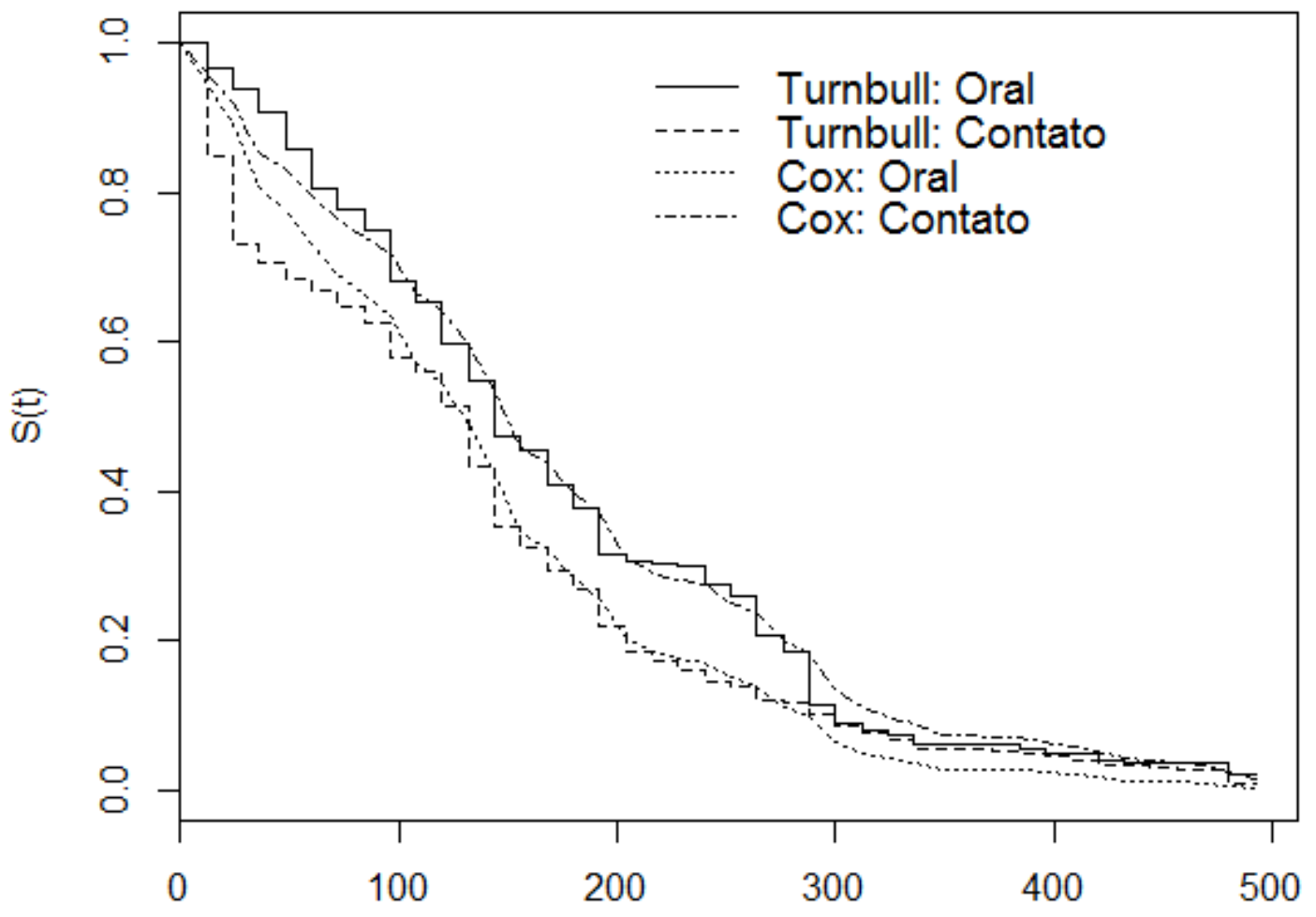

Tempos

Se fosse considerada a análise de variância tradicional o resultado após 48 horas indica diferença entre as vias de intoxicação ( $p=0,0013)$, mas não entre os repelentes $(p=0,5882)$, e nem interação entre os fatores considerados $(p=0,4564)$. Já para o tempo fixo de 96 horas não haveria significância em nenhum dos fatores $(p>0,05)$. Isto evidencia a perda de informação neste tipo de análise.

Atoxicidade oral crônicado repelente causa menordano às abelhas irapuá do que ocontato do mesmo, sendo que o melhor ajuste foi obtido pelo modelo semiparamétrico de Cox, com valores bem próximos aos obtidos pela estimativa de Turnbull.

Assim, independente dos produtos químicos dos repelentes administrados via contato, existe um efeito significativo na sobrevivência da abelha irapuáT. spinipes e recomenda-se que o horário de aplicação do produto seja diferente do horário de forrageamento da T. spinipes, segundo Proni e Marcieira (2004) entre 10 e 15 horas. Isto possibilita traçar estratégias com esses repelentes no controle desse inseto nas culturas de interesse comercial. 0 teste de Log-rank (valor $t=8,73$ ) detectou diferença significativa ( $p=0,003124)$ ao nível de significância $\alpha=0,05$ entre as curvas semiparamétricas referente a intoxicação via oral e por contato.

\section{AGRADECIMENTOS}

Agradecimento ao CNPq e Fapemig pelo apoio financeiro.

\section{REFERÊNCIAS BIBLIOGRÁFICAS}

AGROFIT. Sistemas de agrotóxicos fitossanitários. Disponivel em: http://agrofit.agricultura.gov.br/agrofit cons/principal_agrofit_cons. Acesso: jul 2019.

Archer, C. R.; Pirk, C. W.; Wright, G. A.; Nicolson, S. W. Nutrition affects survival in African honeybees exposed to interacting stressors. Functional ecology, 2014, 28, 913-923.

Barbosa, W. F.; De Meyer, L.; Guedes, R. N. C.; Smagghe, $G$. Lethal and sublethal effects of azadirachtin on the bumblebee Bombus terrestris (Hymenoptera: Apidae). Ecotoxicology, 2015, 24, 1, 130-142. 
Brasil. Ministério da Agricultura, Pecuária e Abastecimento. Página 4 da Seção 1 do Diário Oficial da União (DOU) de 14 de Novembro de 2005. URL (https://www.jusbrasil.com.br/diarios/858452/pg-4secao-1-diario-oficial-da-uniao-dou-de-14-11-2005) (18, jun. 2018)

Brighenti, D. M.; Brighenti, C. R. G.; Carvalho, C. F. Life spans of Africanized honey bees fed sucrose diets enhanced with citric acid or lemon juice. Journal of Apicultural Research, 2017, 56, 2, 91-99.

Boiça Júnior, A. L.; Santos, T. M.; Passilongo, J. Trigona spinipes (Fabr.) (Hymenoptera: Apidae) em espécies de maracujazeiro: flutuação populacional, horário de visitação e danos às flores. Neotropical Entomology, 2004, 135-139.

Boeke, S. J., Boersma, M. G., Alink, G. M., van Loon, J. J., van Huis, A., Dicke, M.; Rietjens, I. M. Safety evaluation of neem (Azadirachta indica) derived pesticides. Journal of ethnopharmacology, 2004, 94 (1), 25-41. URL (https://www.sciencedirect.com/science/ article/pii/S0378874104002491).

Carvalho, L. M.; Bueno, V. H. P.; Almeida, E. F. A. Pragas do copo-de-leite: identificação e danos. Revista Plasticultura, 2009, 4, 17, 26-28.

Chiaradia, L. A; Croce, D. D.; Milanez, J. M.; Morgan, C. Dano e controle da abelha-'irapuá' em eucalipto. Agropecuária Catarinense, 2003, 16, 1,60-62.

Coelho, L. R.; Leonel, S.; Crocomo, W. B.; Labinas, A. M. Controle de pragas do pessegueiro através do ensacamento dos frutos. Ciência e Agrotecnologia, 2008, 32, 1743-1747.

Freitas, B. M.; Pinheiro, J. N. Polinizadores e pesticidas: princípios e manejo para os agroecossistemas brasileiros. Brasília: MMA 2012: 112.

Giolo, S. R.; Colosimo, E. A. Análise de sobrevivência aplicada. Edgard Blucher, 2006. 392 p.

Lin, D. Y. Cox regression analysis of multivariate failure time data: the marginal approach. Statistics in medicine, 1994, 13, 21, 2233-2247.
Maia, M. A.; Eduardo, Y. N. Análise do tempo até a re-hospitalização de pacientes com esquizofrenia via modelo de riscos proporcionais de Cox. Semina: Ciências Exatas e Tecnológicas, 2016, 37, 2, 51-58.

Medrzycki, P., Giffard, H., Aupinel, P., Belzunces, L. P., Chauzat, M. P., Classen, C.; Le Conte, Y. Standard methods for toxicologyresearch in Apis mellifera. Journal of Apicultural Research, 2013, 52, 4, 1-60. URL (https://www.tandfonline.com/doi/ abs/10.3896/IBRA.1.52.4.14)

Melathopoulos, A. P., Winston, M. L., Whittington, R., Smith, T., Lindberg, C., Mukai, A.; Moore, M. Comparative laboratory toxicity of neem pesticides to honey bees (Hymenoptera: Apidae), their mite parasites Varroa jacobsoni (Acari: Varroidae) and Acarapis woodi (Acari: Tarsonemidae), and brood pathogens Paenibacillus larvae and Ascophaera apis. Journal of Economic Entomology, 2000, 93, 2, 199-209. URL (https://academic.oup.com/jee/articleabstract/93/2/199/768002)

Moura, R.; Souza, K; Souza, D.; Santana, G.; Oliveira, G.; Venturoli, F.; Silva-Neto, C. Dano em Khayaivorensis provocado por Trigona spinipes na savana brasileira. Acta Brasiliensis, 2017, 1, 1, 40-42.

Moncharmont, F. X. D.; Decourtye, A.; HennequetHantier, C.; Pons, O.; Pham-Delègue, M. H. Statistical analysis of honeybee survival after chronic exposure to insecticides. Environmental Toxicology and Chemistry. 2003, 22, 12, 3088-3094.

Nisbet, A. J. Azadirachta in from the neem tree Azadirachta indica: its action against insects. Anais da Sociedade Entomológica do Brasil, 2000, 29, 4, 615632.

Pinheiro, J. N.; Freitas, B. M. Efeitos letais dos pesticidas agrícolas sobre polinizadores e perspectivas de manejo para os agroecossistemas brasileiros. Oecologia Australis, 2010, 14, 1, 266-281.

Pirk, C. W.; De Miranda, J. R.; Kramer, M.; Murray, T. E.; Nazzi, F., Shutler, D.; Van Dooremalen, C. Statistical guidelines for Apis mellifera research. Journal of Apicultural Research. 2013, 52, 4, 1-24. 
Previero, C. A.; Junior, B. C. L.; Florêncio, L. K.; Santos, D. L. Receita de plantas com propriedades inseticidas no controle de pragas. CEULP/ULBRA, Palmas, Brasil, 2010. URL(http://www.cnpq.br/ documents/10157/922e31c5-6089-490e-b08095843d86b2b9) (18, jun. 2018)

Proni, E. A.; Macieira, O. J. D. Ritmo circadiano da taxa respiratória de Tetragonisca angustulafiebrigi(Schwarz), T. a. angustula (Latreille) e Trigona spinipes (Fabricius) (Hymenoptera, Apidae, Meliponinae). Revista Brasileira de Zoologia, 2004, 21, 4, 987-993.

Rossi, C. A.; Roat, T. C.; Tavares, D.A; CintraSocolowski, P.; Malaspina, O. Effects of sublethal doses of imidacloprid in malpighian tubules of Africanized Apis mellifera (Hymenoptera, Apidae). Microscopy Research and Technique, 2013, 76, 552-558.

Sanches, A. L.; Slaa, E. J.; Sandi, M.; Salazar, W.; Benedek, P.; Richards, K. W. Use of stingless bees for commercial pollination in enclosures: a promise for the future. Acta Horticulture, 2001, 561, 219-223.
Santos, E. P.; Júnior, C. M. R. S.; Santos, E. M. S.; Santos, H. O.; Costa, K. C. Atuação carrapaticida do neem e manejo consorciado de pastagem no controle do carrapato: revisão sistemática de literatura. Caderno de Ciências Agrárias, 2017, 9, 2, 79-91.

Simões, C.M.O.; Schenkel, E.P.; Gosmann, G.; Mello, J.C.P.; Mentz, L.A.; Petrovick, P.R. Farmacognosia: da planta ao medicamento. 5 ed. Porto Alegre, RS: Ed. da UFSC, 2004.

R, C. T. A language and environment for statistical computing. R Foundation for Statistical Computing, Vienna, Austria.2019. URL(https://www.r-project.org) (19, jun. 2018)

Xavier, V. M., Picanço, M. C., Chediak, M., Júnior, P. A. S., Ramos, R. S., \& Martins, J. C. Acute toxicity and sublethal effects of botanical insecticides to honey bees. Journal of Insect Science, 2015, 15, 1. URL(https://academic.oup.com/jinsectscience/ article/15/1/137/2583443)

Zucchi, R. A.; Silveira Neto, S.; Nakano, O. Guia de identificação de pragas agrícolas. Piracicaba: Fealq, 1993. 